\title{
Isobaric Tags for Relative and Absolute Quantitation (iTRAQ)-Based proteomic analysis of mRNA splicing relevant proteins in aging HSPCs
}

\section{Xiaolan Lian}

Fujian Normal University

Lina Zhang ( $\nabla$ zln_1250@163.com )

shanghai university of traditional chinese medicine https://orcid.org/0000-0001-5804-2252

\section{Research article}

Keywords: iTRAQ, DEPs, HSPC, aging, mRNA splicing

Posted Date: December 19th, 2019

DOI: https://doi.org/10.21203/rs.2.19284/v1

License: (c) (i) This work is licensed under a Creative Commons Attribution 4.0 International License.

Read Full License

Version of Record: A version of this preprint was published at Aging Clinical and Experimental Research on March 5th, 2020. See the published version at https://doi.org/10.1007/s40520-020-01509-z. 


\section{Abstract}

Background: HSPC aging was closely associated with the organism aging, senile diseases and hematopoietic related diseases. Therefore, study on HSPC aging born great significance to further elucidate the mechanisms of aging and to treat hematopoietic disease resulted from HSPC aging. Little attention had been paid to mRNA splicing as a mechanism underlying HSPC senescence.

Results: We used our lab's patented aging model of HSPCs in vitro to analyze mRNA splicing relevant proteins alterations with ITRAQ based proteomic analysis. We found that not only the notable mRNA splicing genes such as SR, hnRNP, WBP11, Sf3b1, Ptbp1 and U2AF1 but also the scarcely reported mRNA splicing relavant genes such as Rbmxl1, Dhx16, Pcbp2, Pabpc1 were significantly down-regulated. We further verified their genes expressions by qRT-PCR. In addition, we reported the effect of Spliceostatin A (SSA), which inhibits mRNA splicing in vivo and in vitro, on HSPC aging.

Conclusions: It was concluded that mRNA splicing emerged as an important vulnerability of HSPC aging. This study improved our understanding of the role of mRNA splicing in the HSPC aging process.

\section{Background}

The mammalian blood system consists of many types of differentiated blood cells such as erythrocytes, T-and B-lymphocytes, myeloid cells. These blood cells are mostly short-lived and need to be replaced depending on hematopoietic progenitor cells (HPCs) and ultimately hematopoietic stem cells (HSCs) [12].

Although blood is the definitive self-renewing tissue of the body, it does not escape the detrimental effects of the aging process. Hematopoietic aging is manifested in human populations in the form of an increase in organism aging and myelopro- liferative disease, including leukemias, declining adaptive immunity, and greater propensity to anemia[3-7].The previous study indicated age-related alterations in the human blood system occur in hematopoietic stem and progenitor cells (HSPCs) [8-9]. Therefore, study on HSPC aging bore great significance to further elucidate the mechanisms of aging and to treat hematopoietic disease resulted from HSC aging.

mRNA splicing is the process by which introns are removed from a pre-mRNA and exons are joined to produce a mature mRNA[10]. A single type of pre-mRNA can be spliced in different ways to generate distinct mRNAs and this process is a major contributor to transcriptomic and proteomic diversity in higher eukaryotes[11].In humans, nearly all multi-exon primary transcripts are alternatively spliced[12-13].

If pre-mRNAs with introns were translated into proteins, truncated and deleterious proteins would be produced since intron sequences harbor a large number of termination codons[14]. 
In recent years, dysregulations of mRNA splicing are emerging to be discovered as important players in organismal ageing, cellular senescence and ageing-related degenerative diseases. Changes in the activity of splicing factors and key splice variants could impact cellular senescence and the aging phenotype[1518]. However, the studies on mRNA splicing mostly focused on a designated gene so far, lack of systematic and comprehensive analysis. Morever, mRNA splicing alterations in HSPC aging process were never reported[19], whether mRNA splicing was an important mechanism of HSPC aging was worth exploring. So, in the present study, we analyzed the alterations of mRNA splicing relevant proteins with iTRAQ based proteomic analysis and Realtime PCR in our lab's patented model of aging HSPCs in vitro, aim to explore the mechanism of mRNA splicing in HSPC aging process. Furthermore, we also observed the effect of Spliceostatin A (SSA), which is a chemically stable, methylated derivative of FR901464, binds to the SF3b complex and inhibits splicing in vivo and in vitro[20],on HSC aging.

\section{Results}

1.Global profiling of proteins in HSPC

The global profiling of proteins in young HSPCs and aging HSPCs were evaluated by the Isobaric Tags for Relative and Absolute Quantitation(iTRAQ) labelling technology in combination with LC-MS/MS. A total of 2542 proteins were identified according to the standard of protein identification (Peptide Threshold 1.0\% FDR, 1 Unique Peptide ) from the four samples(two young group samples and two aging group samples).

2.Differentially expressed proteins(DEPs) between aging HSPCs and young HSPCs

The differentially expressed proteins(DEPs) between aging HSPCs and young HSPCs were selected according to the following criteria: (unique peptide $\geq 2 \& p \otimes 0.01$ ) \&(Fold Change $\otimes 0.67$ or Fold Change $₫ 1.5)$. 302 out of total 2542 proteins were quantified to be differently expressed proteins, including 120 up-regulated proteins and 182 down-regulated proteins. The DEPs were shown as volcano plot (Fig. 1). Furthermore, based on the hierarchical cluster analysis, we also showed the heatmap with the DEPs( Fig. 2).

\section{GO analysis of differentially expressed proteins}

To obtain a global functional view of the DEPs, Gene Ontology (GO) functional classification annotation was conducted with all the DEPs by using GO database (http://www. Geneontology. org/). A GO term was considered significant at $p$-value $<0.05$. The DEPs were categorized by three sets of Ontologies:

$\mathrm{BP}$ (biological process), CC(cellular component), and MF(molecular function). As shown in Fig. 3, the top 5 significantly enriched GO terms in the BP category was cellular metabolic process, metabolic process, organic substance metabolic process, cellular nitrogen compound metabolic process and primary metabolic process. In the CC category, membrane- bounded organelle, extracellular exosome, extracellular vesicle, extracellular organ- elle and organelle were the top 5 significantly enriched terms. For MF, RNA 
binding, binding, heterocyclic compound binding, organic cyclic compound binding and nucleic acid binding were the top 5 significant terms.

It's easy to see from GO data that mRNA splicing was significantly reduced in the aging group compared with the young group ( $p<0.01$; Table $S 1)$. As shown in Table 1 , the DEPs of mRNA splicing were mainly enriched in mRNA splicing via spliceosome, regulation of mRNA splicing via spliceosome,spliceosomal complex assembly, alternative mRNA splicing via spliceosome, regulation of alternative mRNA splicing via spliceosome,mRNA cis splicing via spliceosome,spliceosomal snRNP assembly,positive regulation of mRNA splicing via spliceosome. The key DEPs in these mRNA splicing pathway were shown as Table 1. It might indicate that there was a close relationship between HSPC aging and vulnerability of mRNA splicing.

\section{KEGG pathway analysis of differentially expressed proteins}

To further reveal the potential metabolic pathway involved in HSPCs aging process, signaling pathways analysis of the DEPs was conducted by using the Kyoto Encyclopedia of Genes and Genomes(KEGG) database (http://www. genome.jp/kegg). The result showed that the DEPs were classified into 17 different pathways (Table S2). The top significantly different KEGG pathway was glycolysis, lysosomal, ribosomal synthesis and mRNA splicing(Fig. 4). Glycolysis ( $<<0.01$; Fig.S1) and lysosomal pathway ( $<<$ 0.01 ; Fig.S2) was significantly increased whereas ribosomal synthesis $(p<0.01$; Fig.S3) and mRNA splicing $(p<0.01$; Fig. 5$)$ was significantly reduced in the aging group compare with the young group. We compared and analyzed the DEPs related to mRNA splicing in KEGG database, the results showed that not only the reported well mRNA splicing genes such as SR, hnRNP, WBP11, Sf3b1, U2AF1, Ptbp1 (Fig. 5) but also the possible mRNA splicing relavant genes such as Rbmxl1, Dhx16, Pcbp2, Pabpc1 were significantly down-regulated(Table 1,2). This conclusion is in line with the recent study which showed age-related alterations of splicing factors[21]. This data suggested that mRNA splicing was an important vulnerability of HSPC aging.

\section{Protein-protein interaction analysis}

To further disclose the possible relation between mRNA splicing and HSPC aging, we performed a proteinprotein interaction analysis by STRING Database(http:// string-db.org) version 9.0 with the DEPs of mRNA splicing, aging and hemopoiesis. As seen in Fig. 6, the DEPs of mRNA splicing, aging and hemopoiesis formed a network containing 32 nodes(Fig. 6). The most prominent cluster within the network mainly included splicing proteins. The most important interconnected node was aging related protein Npm1, which had a 2.21-fold down-regulation in response to aging and had the protein-protein interactions with splicing and hemopoiesis. The other noticeable connected nodes were hemopoiesis associated protein Rps19, Rrs1, Ada and Sart3, which had protein- protein interactions with mRNA splicing(Fig. 6).

\section{6.qRT-PCR}


To validate the accuracy of the quantitative proteomic analysis, $8 \mathrm{mRNA}$ splicing related genes were randomly selected for realtime-PCR analysis. The A260/280 ratio of RNA extracted from HSPCs was 1.81.9 , indicating high purity of RNA.The result showed that the mRNA levels of mRNA splicing related genes including Rbmxl 1, Dhx16, Wbp11, U2af1, U2af2, Pcbp2, Ptbp1, Pabpc1 verified by qRT-PCR had the similar trends to the protein levels tested by the quantitative proteomic analysis. The fold changes of transcript abundances are provided in Fig. 7. It suggested the proteomic analysis was accurate.

\section{Effect of SSA treatment on the aging of HSPC}

Spliceostatin A (SSA) (Fig. 8)is a chemically stable, methylated derivative of FR901464, can inhibits mRNA splicing in vivo and in vitro. To verify the important role of mRNA splicing in the HSPC aging process, we treated HSPCs with SSA and observed the effect of SSA treatment on aging-related manifestations of HSPCs with SA- $\beta$-gal staining and Mixed colony-forming unit (CFU-Mix) culture Method.SA- $\beta$-gal (Senescence-associated- $\beta$-galactosidase) is a hallmark of aging that can yield a blue stain in the cytoplasm of aging cells. We found that the percentage of SA- $\beta$-gal stain-positive in SSA group(HSPCs were cultured with the culture medium (STEMCELL Technologies,) supplemented with the addition of $100 \mathrm{ng} \mathrm{mL}-1$ of SSA for $72 \mathrm{~h}$,Fig. 9B ) was significantly higher than the control group(HSPCs were cultured with the culture medium for $72 \mathrm{~h}$, Fig. 9A )). The mixed colony-forming units of the HSPCs in SSA group were less than the control group(Fig. 10). We speculated that the inhibitor of mRNA splicing result in HSPCs aging, it further proved the hypothesis that mRNA splicing was an important mechanism of HSPC aging.

\section{Discussion}

Little attention has been paid to mRNA splicing as a mechanism underlying SC/HSPC senescence. RNA splicing is highly regulated in higher eukaryotic cells, performed by spliceosome, a large nuclear macromolecular complex that contains five small nuclear ribonucleoproteins (snRNPs) (U1, U2, U4, U5 and U6) and more than 150 accessory proteins [21-24]. These accessory proteins include SR protein (serine/arginine-rich protein), hnRNP(heterogeneous nuclear ribonucleoprotein,hnRNP), RNA helicase,

et al[25].SR protein contain at least one RNA recognition motif and an arginine serine-rich domain to bind the pre-mRNA, regulating splicing and alternative splicing in a concentration-dependent manner. hnRNP, instead, influence splicing events by preferentially binding to splicing silencers[26].Studies of mammalian splicing factors are often focused on snRNP or regulatory RNA-binding proteins, such as hnRNP and SR proteins, however, much less is known about the contribution of RNA helicases. About splicing factor of RNA helicase, DExD/H-box proteins are concerned and they are a family of RNA-dependent ATPases (or NTPases), which utilize the energy from ATP hydrolysis to modulate the structure of RNA or ribonucleoprotein complexes [27].

The present study showed that not only the notable mRNA splicing genes such as SR, hnRNP, snRNPs, RNA helicase but also the mRNA splicing relavant gene such as Wbp11, u2af, Rbmxl1, Pcbp2, Pabpc1, thrap3, Raver1 and snu13 were significantly downregulated in aging HSPC. By combining ITRAQ data and Uniprot database, we focus on 4 notable mRNA splicing genes Wbp11, u2af1, u2af2, Ptbp1 and 4 
scarcely reported mRNA splicing relavant genes Rbmxl1,Dhx16, Pcbp2,Pabpc1, which were verified by qRT-PCR.

Pabpc1(Polyadenylate-binding protein 1,PABP-1) is the component of mRNP(mRNA ribonucleoprotein) complex which is at least composed of DHX9, DDX3X, ELAVL1, HNRNPU, IGF2BP1, ILF3, PABPC1, PCBP2, PTBP2, STAU1, STAU2, SYNCRIP and YBX1[28]. It serves as a central regulator of mRNA fate, mRNA utilization and destruction [29-30].Pabpc1 may be involved in cytoplasmic regulatory processes of mRNA metabolism such as pre-mRNA splicing, but need to be confirmed. Pcbp2(Poly rC-binding protein 2 ), is a Single-stranded nucleic acid binding protein that binds preferentially to oligo dC. It is identified as a component of mRNP complex. Cong Ren,et al showed that Pcbp2 regulates p73-dependent biological function in ROS production and cellular senescence[31].Rbmxl1 (RNA binding motif protein, X-linked-like1)is also be known as heterogeneous nuclear ribonucleoprotein G-like 1. Gene burden analysis revealed three genes associated with extreme aging, including Rbmxl1[32]. These data suggested a possible close link among Pcbp2, Rbmxl1 and aging, thus it is unclear what is the roles of Pcbp2/Rbmxl1 in HSC aging process. About splicing factor of RNA helicase, Dhx16(ATP-dependent RNA helicase DHX16), is an ATPase that activates the spliceosome before the first catalytic step of splicing[33]. Gencheva M, et al, further suggest that mutant DHX16 causes a defective spliceosome to retain unspliced gene transcripts in the nuclei of human cells[34].It indicated DHX16 was involved in mRNA splicing, however, whether Dhx16 affects aging is largely unknown.

The most important interconnected node rotein in String analysis, Nucleophosmin (NPM), also known as B23, numatrin and NO38, is a multi- functional protein. It's involved in diverse cellular processes such as ribosome biogenesis, centrosome duplication, protein chaperoning, histone assembly, cell proliferation, and regulation of tumor suppressors p53/TP53 and ARF[35].Luiza Handschuh's study indicated that aberrant proportions of particular NPM1 transcripts could be linked to abnormal expression of genes encoding alternative splicing (AS) factors.It indicated NPM1 was involved in mRNA splicing, however, whether NPM1 affects aging was unknown.

It was suggested from this study that Rbmxl1, Dhx16, Pcbp2, Pabpc1and NPM screened by the prestent study may play an important role in the process of HSPC aging. In addition, HSPCs treated with SSA, the inhibitor of mRNA splicing, showed the manifestations of cell senescence, further proved the hypothesis that mRNA splicing was an important mechanism of HSPC aging. This, we had reasoned, would provide the experimental support for researching mRNA splicing in HSC aging.

\section{Conclusion}

Important mRNA splicing relavant genes, including SR, hnRNP, WBP11, Sf3b1, U2AF1, Ptbp1, Rbmxl1, Dhx16, Pcbp2, Pabpc1 were compromised by HSPC aging. String network analysis suggested that NPM1 were the most important protein regulation hubs of HSPC aging and mRNA splicing.HSPCs treated with SSA, the inhibitor of mRNA splicing, showed the features of cell senescenc proved the hypothesis that mRNA splicing was an important mechanism of HSPC aging.The identification of these mRNA splicing 
associated proteins can help us to better understand the molecular mechanisms of mRNA splicing in HSPC aging.

\section{Methods}

Animals

Equal numbers of male and female C57BL/6J SPF mice were obtained from Shanghai Sippr-BK Experimental Animal Center [Certificate No. SCXK (Shanghai) 2013- 0016]. The mice were 4 weeks of age and $16-18 \mathrm{~g}$ in weight, no gender limitation.

Chemicals and reagents

Red blood cell lysis buffer was purchased from Beyotime Biotechnology Co., Ltd. Anti-c-kit (CD117) MicroBead and Lineage Cell Depletion kits were purchased from Miltenyi Co., Ltd. RNA Extraction and Purification kits, Reverse Transcription and Fluorescence Quantitative PCR kits were purchased from Takara Co., Ltd. Stemspan Stem Cell Media were purchased from Stem cell Co., Ltd. Mouse ColonyForming Unit (CFU-Mix) Assays Using MethoCult ${ }^{\text {TM }}$ were purchased from Stem cell Co., SA- $\beta$-Gal staining kit and cell cycle detection kit were purchased from Beyotime Biotechnology Co.

Sample Treatment and Sequencing

1. Isolation and Purification of HSPCs

4 weeks-old mice were both euthanatized by chloral hydrate, and were sacrificed with total bone-marrow cells isolated from femurs and tibiae. All animal experiments were performed in compliance with the guidelines of the Animal Care and Use Committee of SHUTCM(Shanghai Universitiy of Traditional Chinese Medicine).The cells was suspended in red blood cell lysis buffer(Beyotime Biotechnology Co., Ltd), incubated at room temperature for $5 \mathrm{~min}$, then it was centrifuged at $3000 \mathrm{rpm}$ for $5 \mathrm{~min}$ and the supernatant was discarded. The precipitate was washed again, what we get was bone marrow mononuclear cells (MNCs). These cells were suspended in PBS containing EDTA and 0.5\% BSA. Then we obtained HSC/HPCs through $\mathrm{Lin}^{-}{ }^{-} \mathrm{ckit}^{+}$immunomagnetic bead sorting with lineage cell depletion kits (Miltenyi Co.) and anti-c-Kit microbead (Miltenyi Co.)[36].

2. Modeling and Identification of the Aging HSPC Model in vitro

1) Young HSPC group: Repeat the procedure obove with 4 week-old mice, $n=10$

2) Old Model HSPC group: Young group HSPCs were cultured with stem cell culture medium (STEMCELL Technologies) $+10 \mathrm{ng} / \mathrm{ml} \mathrm{IL3}+10 \mathrm{ng} / \mathrm{ml} \mathrm{IL} 6+30 \mathrm{ng} / \mathrm{ml} \mathrm{SCF}$ and incubated at $37{ }^{\circ} \mathrm{C}, 5 \% \mathrm{CO}_{2}$ for 8 days. The medium was changed every 2 to 3 days. $n=10$.

The details for identification of the model was in our patented method: a method to establish old HSPC model in vitro. 


\section{Protein Extraction and Quantification}

Lysis buffer (2\% SDS, 7M urea, $1 x$ Protease Inhibitor Cocktail) was added into the samples $(n=3)$. The lysis was performed by sonication on ice for $3 \mathrm{~min}$ and kept on ice for $30 \mathrm{~min}$. After centrifugation at $15000 \mathrm{rpm}$ for $15 \mathrm{~min}$ at $4^{\circ} \mathrm{C}$, the supernatant was collected and transferred to a new Eppendorf tube. An aliquot of the supernatant was taken and the protein concentration was determined by Bio-Rad protein assay (Bio- Rad, Hercules, CA, USA).

4. Isobaric tags for relative and absolute quantification (iTRAQ)

Protein digestion and iTRAQ labeling were performed according to the related literatures. Briefly, 100 ug of protein from each sample was reduced, alkylated and digested with sequence-grade modified trypsin (Promega, Madison, WI) prior to labeling with one of the individual 8-plex-iTRAQ tags (Applied Biosystems, Framingham, MA). The peptide mixture was fractionated by high $\mathrm{pH}$ separation using Ultimate 3000 system (Thermo Fisher scientific, MA, USA) connected to a reverse phase column (XBridge C18 column, $4.6 \mathrm{~mm} \times 250 \mathrm{~mm}, 5 \mu \mathrm{m}$, Waters Corporation, MA, USA). Twelve fractions were separated by nanoLC and analyzed by on-line electrospray tandem mass spectrometry. The experiments were performed on an Easy-nLC 1000 system (Thermo Fisher Scientific, MA, USA) connected to a Q-Exactive mass spectrometer (Thermo Fisher Scientific, MA, USA) equipped with an online nano-electrospray ion source. PEAKS DB was set up to search the Uniprot-mouse database (ver.201711, 51946 entries) assuming the digestion enzyme Trypsin.

5. Identification of Differentially Abundant Proteins Protein identification and quantification was performed using the Mascot 2.3.02 search engine against the UniProt database (http://www.uniprot.org). To demonstrate the repeatability of the replicates, protein abundances between various biological replicates were compared.

6. Go, KEGG and STRING Enrichment Analyses

In order to obtain a global functional view of the DEPs, Gene Ontology (GO) func- tional classification annotation and Kyoto Encyclopedia of Genes and Genomes (KEGG) metabolic pathway analysis were conducted. All the DEPs were subjected to GO analysis(http://www. geneontology.org) and KEGG analysis (http ://www. Gen ome.jp/kegg). GO database is an internationally standardized gene functional classi-fication system that comprehensively describes characteristics of genes and their products. KEGG was used to predict the major metabolic and signal transduction pathways involved in DEPs[37]. Furthermore, to study the potential roles of mRNA splicing in HSPC aging,we built a regulatory network with the DEPs using STRING analysis(http://www.string -db.org/)[38].

7. qRT-PCR

To investigate whether the differences in protein abundances were reflected at the mRNA level and to validate the proteomic data, the quantitative real-time polymerase chain reaction(qRT-PCR) was used to verify the mRNA level of mRNA splicing associated proteins between the young group and the aging group. 4 notable mRNA splicing genes Wbp11, u2af1, u2af2,Ptbp1 and 4 scarcely reported mRNA splicing relavant genes Rbmxl1,Dhx16, Pcbp2,Pabpc1 in HSPC were selected for qRT-PCR analysis to validate the accuracy of the ITRAQ proteomic analysis. Total RNA extraction and reverse transcription were performed according to the manufacturer's instructions for the kit used (9108/9109; RR047A, Takara). The A260/280 ratio of RNA was dete- cted.The primers were designed and synthesized by sango biotech. All 
primer sequences are listed in Table 3. The GAPDH (internal control) primers were used. SYBR green dye was used for Real-time quantitative PCR (RR420A, Takara). The $2^{-\Delta \Delta C t}$ method was used to calculate mRNA expression levels. $\Delta \mathrm{Ct}=\mathrm{Ct}_{\text {target }}$ gene $-\mathrm{Ct}_{\text {internal }}$ control gene (where $\mathrm{Ct}$ is the cycle number when the fluorescence signal reaches the set threshold). The amplification parameters were: $95^{\circ} \mathrm{C}$ for $30 \mathrm{~s},\left(95^{\circ} \mathrm{C}\right.$ for $5 \mathrm{~s}, 60^{\circ} \mathrm{C}$ for $34 \mathrm{~s}$ ) for 40 cycles. The analysis was performed with three biological replicates.

8. Detection of HSPC aging related manifestation after SSA treatment

SA- $\beta$-gal staining

HSPCs( 1000,000 cells) were collected on $72 \mathrm{~h}$ after the treatment with $100 \mathrm{ng} \mathrm{mL}-1$ of SSA. The cells were fixed with $4 \%$ paraformaldehyde for 15 minutes, then incubated at $37^{\circ} \mathrm{C}$ without $\mathrm{CO}_{2}$ for $16 \mathrm{~h}$ in $\beta$ galactosidase staining solution. The number of $\beta$-galactosidase positive cells per 400 total cells was counted, $\mathrm{n}=3$.

Mixed colony-forming unit (CFU-Mix) of HSPC culture

HSPCs ( 1000,00cells) were collected and subjected to mixed colony-forming unit (CFU-Mix) culture: Cells were diluted, in duplicate, with IMDM $+2 \%$ FBS and MethoCult ${ }^{\text {TM }}$ GF M3434 medium to a final concentration of $5 \times 10^{3}$ per $35 \mathrm{~mm}$ dish. $0.3 \mathrm{~mL}$ of the diluted cells were added to $3 \mathrm{~mL}$ of MethoCult $\mathrm{t}^{\mathrm{TM}}$ and mixed thoroughly. The final cell mixture was dispensed into each $35 \mathrm{~mm}$ dish at a volume of $1.1 \mathrm{~mL}$ and incubated at $37^{\circ} \mathrm{C}$ in $5 \% \mathrm{CO}_{2}$ for 7 days. Photos were taken using an inverted microscope. Finally, we added $1 \mathrm{mg} / \mathrm{ml} \mathrm{p}$-iodonitrotetrazolium violet to 24-well cells so as to take photos 24 hours later. The number of CFU-Mix per $5 \times 10^{3}$ cells represented the pluripotency of the HSPCs, $n=3$.

Statistical analysis

The experimental data was expressed as a mean and standard deviation. Single factor analysis of variance and one-way ANOVA was performed using SPSS 18.0. The LSD or Tamhane test was used to compare differences between two groups. $p<0.05$ was considered statistically significant.

\section{Abbreviataions}

Isobaric Tags for Relative and Absolute Quantitation ITRAQ

DEPs Differentially Expression Proteins DEPs

hematopoietic stem / progenitor cells HSPC

bone marrow mononuclear cells MNCs

Gene Ontology GO

Kyoto Encyclopedia of Genes and Genomes KEGG

Spliceostatin A SSA

\section{Declarations}

Ethics approval and consent to participate 
All animal experiments were approved by the Animal experimental ethics committee of Shanghai university of Traditional Chinese Medicine, and were carried out in accordance with the institutional guidelines and ethics.

Consent for publication

All authors of this article have consented for publication.

Availability of data and materials

The datasets used and/or analysed during the current study are available from the corresponding author on reasonable request.

Competing Interests

The authors declare no conflict of interest exists.

Funding

This work was financially supported by National Science Fund of China (No. 81403279). The funding sponsors had no role in the design of the study and collection, analysis, and interpretation of data and in writing the manuscript.

Author Contributions

NZ conceived and designed the research. LL conducted the experiments. All authors have read and approved the manuscript.

Acknowledgment

Not Applicable.

\section{References}

1. Akunuru S, Geiger H. Aging, Clonality, and Rejuvenation of Hematopoietic Stem Cells. Trends Mol. Med.2016, 22, 701-712

2. Moehrle BM,Geiger $\mathrm{H}$. Aging of hematopoietic stem cells: DNA damage and mutations? Exp. Hematol.2016, 44, 895-901.

3. de Haan G, Lazare SS. Aging of hematopoietic stem cells.Blood. 2018,1;131(5):479-487

4. Chung SS, Park CY.Aging, hematopoiesis, and the myelodysplastic syndromes. Hematology Am Soc Hematol Educ Program. 2017(1):73-78 
5.Ren R, Ocampo A, Liu GH, et al.Regulation of Stem Cell Aging by Metabolism and Epigenetics.Cell Metab. 2017 Sep 5;26(3):460-474.

6.Waterstrat A, Van Zant G.Effects of aging on hematopoietic stem and progenitor cells.Cell Metab. 2017 ,26(3):460-474

7. Warren LA, Rossi DJ.Stem cells and aging in the hematopoietic system.Mech Ageing Dev. 2009,130 (12):46-53.

8.Liran I. Shlush. Age-related clonal hematopoiesis. Blood,2018,131:496-504

9.Lee J, Yoon SR, Choi I, et al. Causes and Mechanisms of Hematopoietic Stem Cell Aging.Int J Mol Sci,2019,20(6):13

10.Sahebi M, Hanafı MM, van Wijnen AJ,et al. Towards understanding pre-mRNA splicing mechanisms and the role of SR proteins.Gene. 2016, 587(2):107-19

11.Lulzim Shkreta, Benoit Chabot.The RNA Splicing Response to DNA Damage. Biomolecules, 2015, 5:2935-2977

12.Pan Q,Shai O,Lee LJ,et al. Deep surveying of alternative splicingcomplexity in the human transcriptome by high-throughput sequencing. Nat Genet. 2008, 40:1413-1415.

13.Wang ET, Sandberg R, Luo S, et al. Alternative isoform regulation in human tissue transcriptomes. Nature,2008, 456:470-476

14.Yoshimoto R,Kaida D,Furuno M,et al.Global analysis of pre-mRNA subcellular localization following splicing inhibition by spliceostatin A. RNA. 2017,23(1): 47-57

15.Mathieu Deschenes, Benoit Chabot.The emerging role of alternative splicing in senescence and aging.Aging Cell, 2017,16, pp918-933

16.Daguenet E, Dujardin G, Valcárcel J.The pathogenicity of splicing defects: mechanistic insights into pre-mRNA processing inform novel therapeutic approaches. EMBO Rep,2015 16(12): 1640-55.

17.Scotti MM, Swanson MS. RNA mis-splicing in disease.Nat Rev Genet., 2016,17(1):19-32

18.Chabot B, Shkreta L.Defective control of pre-messenger RNA splicing in human disease. J Cell Biol, 2016, 212(1):13-27

19.Moehrle BM, Geiger H.Aging of hematopoietic stem cells: DNA damage and mutations? Exp Hematol, 2016, 44(10):895-901

20.Roybal GA,Jurica MS. Spliceostatin A inhibits spliceosome assembly subsequent to prespliceosome formation.Nucleic Acids Res. 2010,38(19):6664-72. 
21.Mathieu Deschenes, Benoit Chabot. The emerging role of alternative splicing in senescence and aging. Aging Cel,3017,16, pp918-933

22.Matera AG,Wang Z. A day in the life of the spliceosome. Nat. Rev. Mol. Cell Biol,2014, 15:108-121

23.Wahl MC,Luhrmann R. SnapShot: Spliceosome dynamics II. Cell ,2015, 162(2):456-456

24.Yan C, Wan R, Shi Y.Molecular Mechanisms of pre-mRNA Splicing through Structural Biology of the Spliceosome.Cold Spring Harb Perspect Biol. 2019,11(1). pii: a032409

25.Guil S, Gattoni R, Carrascal M, et al.Roles of hnRNP A1, SR proteins, and p68 helicase in c-H-ras alternative splicing regulation.Mol Cell Biol, 2003,23(8):2927-41.

26.Kędzierska H, Piekiełko-Witkowska A. Splicing factors of SR and hnRNP families as regulators of apoptosis in cancer.Cancer Lett, 2017,$28 ; 396: 53-65$

27.Liu YC, Cheng SC. Functional roles of DExD/H-box RNA helicases in Pre-mRNA splicing. J Biomed Sci.,2015,22:54

28.Safaee N, Kozlov G, Noronha AM, et al. Interdomain allostery promotes assembly of the poly(A) mRNA complex with PABP and elF4G. Mol Cell. 2012,48(3):375-86.

29.Burgess HM, Gray NK. mRNA-specific regulation of translation by poly(A)-binding proteins. Biochem Soc Trans. 2010,38:1517-1522.

30.Gorgoni B, Richardson WA, Burgess HM, et al. Poly(A)-binding proteins are functionally distinct and have essential roles during vertebrate development. Proc Natl Acad Sci USA. 2011,108:7844-7849.

31.Cong Ren,Jin Zhang,Wensheng Yan, et al. RNA-binding Protein PCBP2 Regulates p73 Expression and p73-dependent Antioxidant Defense. J Biol Chem. 2016,291(18): 9629-9637.

32.Nygaard HB, Erson-Omay EZ, Wu X, et al.Whole Exome Sequencing of an Exceptional Longevity Cohort. J Gerontol A Biol Sci Med Sci. 2019,74(9):1386-1390

33. Gencheva M, Kato M, Newo AN, et al.Contribution of DEAH-box protein DHX16 in human pre-mRNA splicing. Biochem J. 2010,429(1):25-32

34. Gencheva M, Lin TY, Wu X, et al. Nuclear retention of unspliced pre-mRNAs by mutant DHX16/hPRP2, a spliceosomal DEAH-box protein.J Biol Chem. 2010,285(46):35624-32.

35. Handschuh L, Wojciechowski P, Kazmierczak M, et al. NPM1 alternative transcripts are upregulated in acute myeloid and lymphoblastic leukemia and their expression level affects patient outcome.J Transl Med. 2018,16(1):232 
36.Lian X, Dong Y, Zhao M, Liang Y, Jiang W, Li W, Zhang L.RNA-Seq analysis of differentially expressed genes relevant to mismatch repair in aging hematopoietic stem-progenitor cells. J Cell Biochem. 2019, 25. doi: $10.1002 /$ jcb.28417

37.Kanehisa M, Goto S, Sato Y, et al. Data, information, knowledgeand principle: Back to metabolism in KEGG. Nucleic Acids Res. 2014, 42, D199-D205

38.Szklarczyk D,Franceschini A,Wyder S,Forslund K,Heller D,Huerta-Cepas J, Simonovic M, Roth A, Santos A, Tsafou K, et al. STRING v10: Protein-protein interaction networks, integrated over the tree oflife. Nucleic Acids Res. 2015, 43, D447-D452.

\section{Tables}




\begin{tabular}{|c|c|c|c|}
\hline Number & Pathway Name & Mismatch Repair Related Genes & $\begin{array}{l}\mathrm{P} \\
\text { Value }\end{array}$ \\
\hline 1 & $\begin{array}{l}\text { mRNA splicing, } \\
\text { via spliceosome }\end{array}$ & $\begin{array}{l}\text { Hnrnpa2b1, Pabpc1, Sf3b6, Snrpg, Snrpd3, Dhx16, Thrap3, } \\
\text { Npm1,Pcbp2, Srsf2, Srsf1, U2af2, Ptbp1, Srsf7, Hnrnpr, } \\
\text { Rbmxl1, Rbm5, Wbp11, Raver1, Snu13, U2af1, Dcps, Sart3 }\end{array}$ & $\begin{array}{l}1.35 \mathrm{E}- \\
11\end{array}$ \\
\hline 2 & $\begin{array}{l}\text { regulation of } \\
\text { mRNA splicing, } \\
\text { via spliceosome }\end{array}$ & $\begin{array}{l}\text { Hnrnpa2b1, Thrap3, Npm1, Srsf2, U2af2, Ptbp1, Srsf7, } \\
\text { Rbmxl1, Rbm5 }\end{array}$ & $\begin{array}{l}2.99 \mathrm{E}- \\
06\end{array}$ \\
\hline 3 & $\begin{array}{l}\text { spliceosomal } \\
\text { complex } \\
\text { assembly }\end{array}$ & Snrpg, Snrpd3(snRNP), Srsf1, Rbm5, Sart3, Rbmxl1 & $\begin{array}{l}7.32 \mathrm{E}- \\
05\end{array}$ \\
\hline 4 & $\begin{array}{l}\text { alternative mRNA } \\
\text { splicing, via } \\
\text { spliceosome }\end{array}$ & Thrap3, Srsf2, Srsf1, Ptbp1, Rbmxl1, Rbm5 & $\begin{array}{l}1.90 \mathrm{E}- \\
04\end{array}$ \\
\hline 5 & $\begin{array}{l}\text { regulation of } \\
\text { alternative mRNA } \\
\text { splicing, via } \\
\text { spliceosome }\end{array}$ & Thrap3, Srsf2, Ptbp1(hnRNP1), Rbmxl1, Rbm5 & $\begin{array}{l}6.05 \mathrm{E}- \\
04\end{array}$ \\
\hline 6 & $\begin{array}{l}\text { mRNA cis splicing, } \\
\text { via spliceosome }\end{array}$ & Srsf1, Wbp11, Dcps & $\begin{array}{l}6.15 \mathrm{E}- \\
04\end{array}$ \\
\hline 7 & $\begin{array}{l}\text { spliceosomal } \\
\text { snRNP assembly }\end{array}$ & Snrpg, Snrpd3, Sart3 & $\begin{array}{l}8.56 \mathrm{E}- \\
3\end{array}$ \\
\hline 8 & $\begin{array}{l}\text { positive regulation } \\
\text { of mRNA splicing, } \\
\text { via spliceosome }\end{array}$ & Thrap3, Rbmxl1 & $\begin{array}{l}2.38 \mathrm{E}- \\
2\end{array}$ \\
\hline
\end{tabular}




\begin{tabular}{|lllll|}
\hline \multicolumn{5}{|l}{ Table2 KEGG Differential Expressions of } \\
mRNA splicing Associated proteins in HSPCs(Aging/Young) \\
\cline { 1 - 4 } Gene name & Geneic expression level & Fold change & P value \\
\cline { 2 - 4 } & OID Group & Control Group & & \\
\cline { 1 - 4 } Snrpg & 1996000 & 3746000 & -1.88 & $4.36 \mathrm{E}-4$ \\
\hline Srsf1 & 3708000 & 6015000 & -1.62 & $6.97 \mathrm{E}-3$ \\
\hline Rbmxl1 & 397100 & 681500 & -1.72 & $9.16 \mathrm{E}-4$ \\
\hline Srsf5 & 1036000 & 1726000 & -1.67 & $4.01 \mathrm{E}-3$ \\
\hline Wbp11 & 274900 & 461200 & -1.68 & $5.84 \mathrm{E}-3$ \\
\hline Srsf2 & 2602000 & 5057000 & -1.94 & $2.36 \mathrm{E}-3$ \\
\hline Sf3b6 & 488500 & 918900 & -1.88 & $7.78 \mathrm{E}-3$ \\
\hline Srsf7 & 2093000 & 4353000 & -2.08 & $1.58 \mathrm{E}-4$ \\
\hline Snrpd3 & 687800 & 1122000 & -1.63 & $9.84 \mathrm{E}-3$ \\
\hline U2af1 & 914100 & 1846000 & -2.02 & $5.88 \mathrm{E}-4$ \\
\hline Snu13 & 1638000 & 3252000 & -1.99 & $7.24 \mathrm{E}-3$ \\
\hline
\end{tabular}




\begin{tabular}{|c|c|c|}
\hline \multirow[t]{2}{*}{ Primers } & Genbank Reference/ & Fragment Length \\
\hline & Sequence & \\
\hline Actin (forward) & GTACCACCATGTACCCAGGC & $247 \mathrm{bp}$ \\
\hline Actin (reverse) & AACGCAGCTCAGTAACAGTCC & \\
\hline Rbmxl 1(forward) & AGGAGGAAGTGGAGGAACTAGG & $179 \mathrm{bp}$ \\
\hline Rbmxl 1 (reverse) & TTCGAACTGGTCCGGAAGGT & \\
\hline Wbp11(forward) & AGGAATCCGAGGGCCTTTAC & $293 \mathrm{bp}$ \\
\hline Wbp11(reverse) & CCTTCGGTCGCTGAATGAGG & \\
\hline U2af1(forward) & CCTTTAGCCAGACCATTGCC & $188 \mathrm{bp}$ \\
\hline U2af1(reverse) & GTCGCAGACGTTCATCTCCT & \\
\hline U2af2(forward) & AAGCGTAGTCACAGTCGCTC & $155 \mathrm{bp}$ \\
\hline U2af2 (reverse) & АTCAATCCACCGTGCTCCTC & \\
\hline Pcbp2(forward) & AGGGGATATGCTCCCСАACT & $103 b p$ \\
\hline Pcbp2(reverse) & CTCCAACATGACCACGCAGA & \\
\hline Ptbp1(forward) & CTGCAGTATGCTGACCCTGT & $252 b p$ \\
\hline Ptbp1(reverse) & TGCATACGGAGAGGCTGACA & \\
\hline Dhx16(forward) & CGGCCCAAGGATAAGGTTGT & $215 b p$ \\
\hline Dhx16(reverse) & ACTTCCACACGCTCCAAGAG & \\
\hline Pabpc1(forward) & CAGGGCAAAGGAGTTCACCA & $275 b p$ \\
\hline Pabpc1(reverse) & TAAGTTCCGTCTGCCGTTCC & \\
\hline
\end{tabular}

\section{Figures}




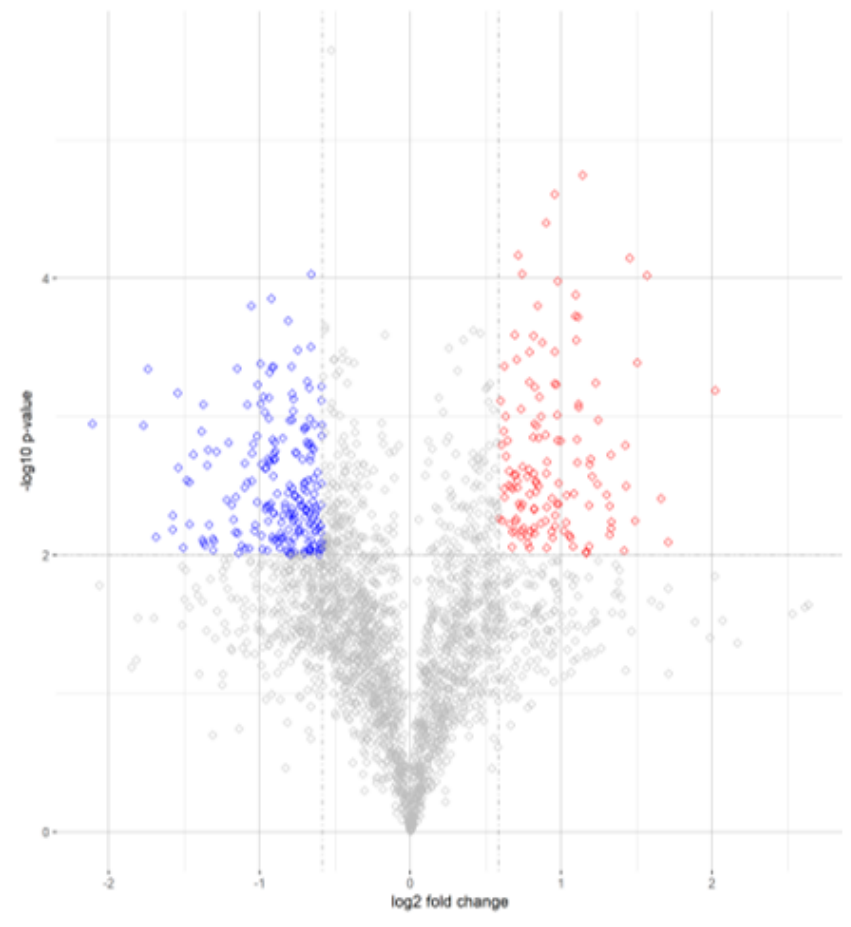

Fig.1 A

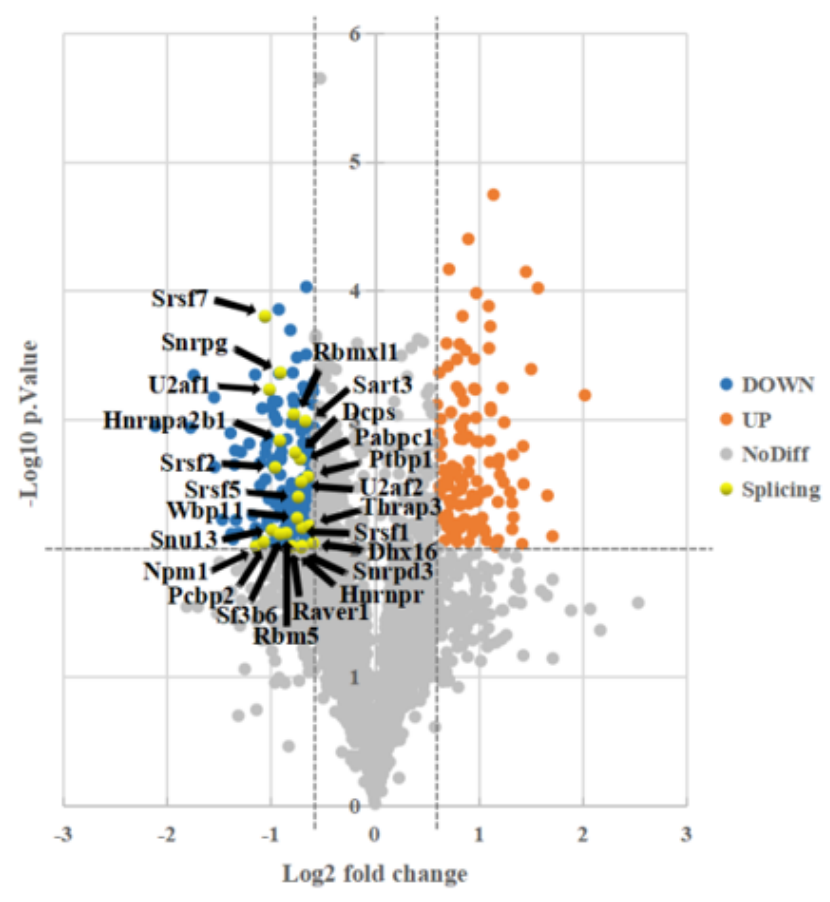

Fig.1 B

\section{Figure 1}

The Volcano plot of the DEPs(Differently expressed proteins) (A). Each point represents the difference of protein expression (fold-change) between two groups. Red point represents upregulated protein; blue point represents downregulated protein. Gray point represents no significant differential protein. The Xaxis represent -s log2-fold change; The Y-axis corresponds to -log10-P value.(B); mRNA splicing relevant DEPs were marked in the Volcano plot. 


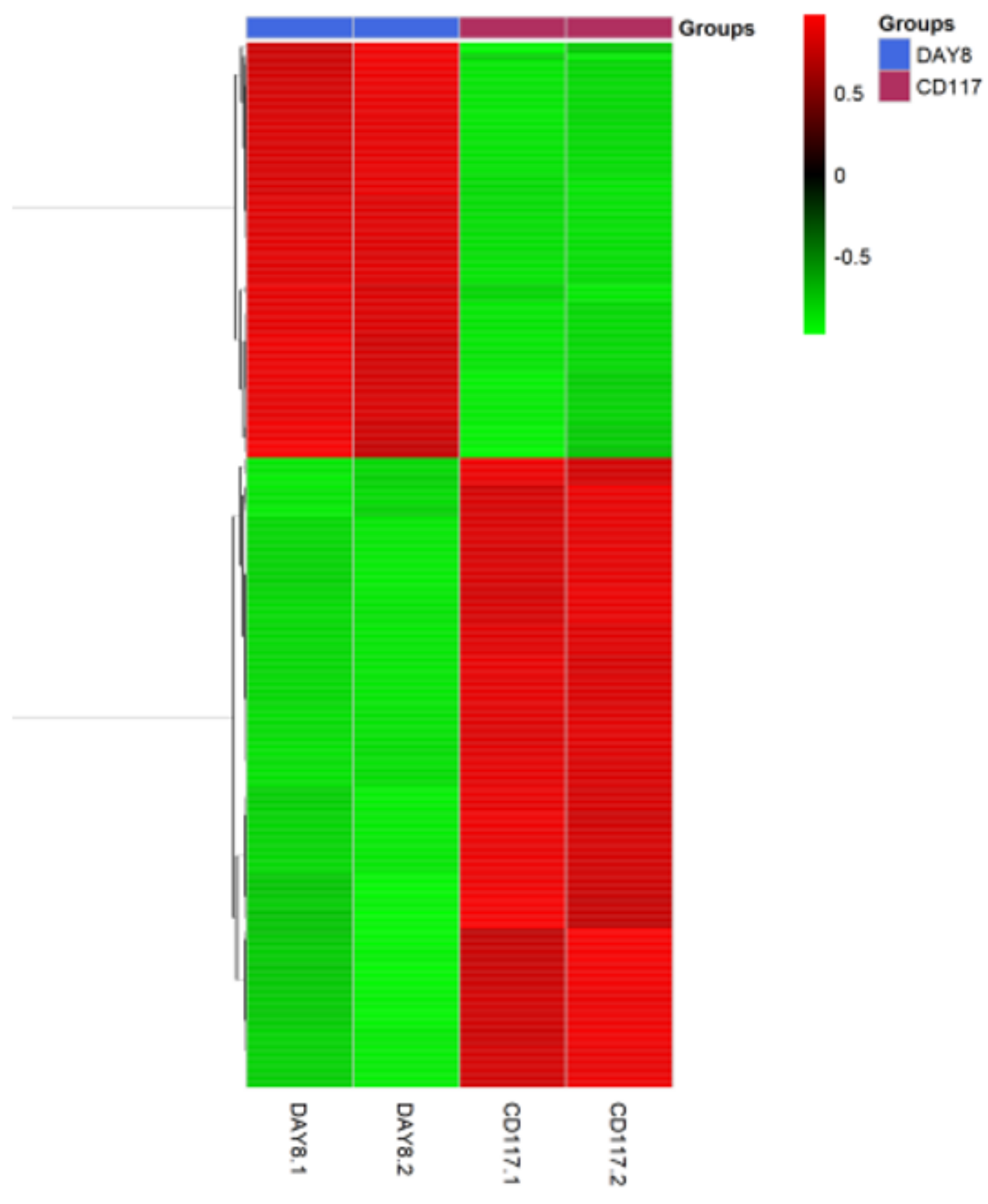

Figure 2

Hierarchical cluster analysis of DEPs Heatmap analysis is an algorithmic approach to find discrete groups with varying degrees of (dis)similarity in a data set represented by a (dis)similarity matrix and is processed with pheatmap package (https://CRAN.Rproject. org/ package= pheatmap). Green signifies down-regulated proteins and red signifies up-regulated proteins in aging group compared with young HSPCs. 


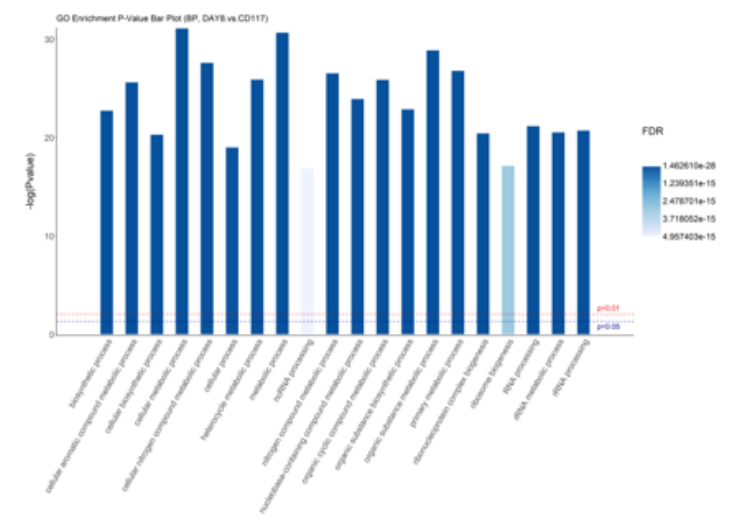

Fig.3A

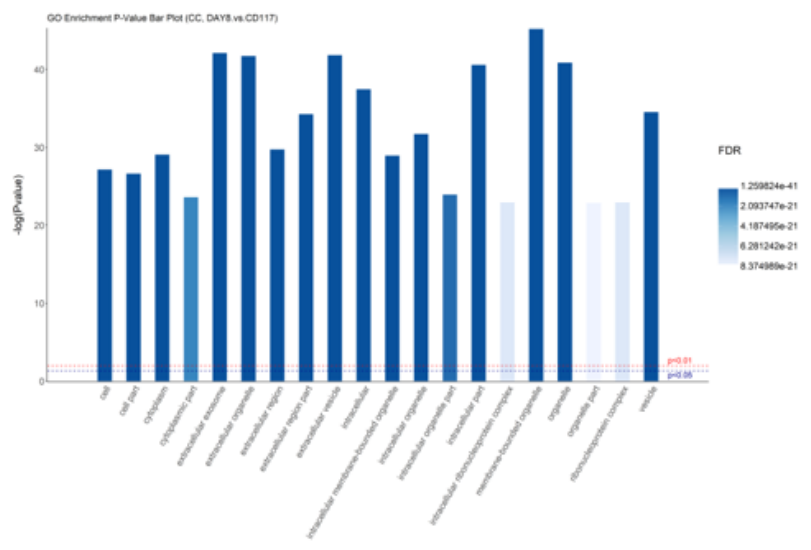

Fig.3B

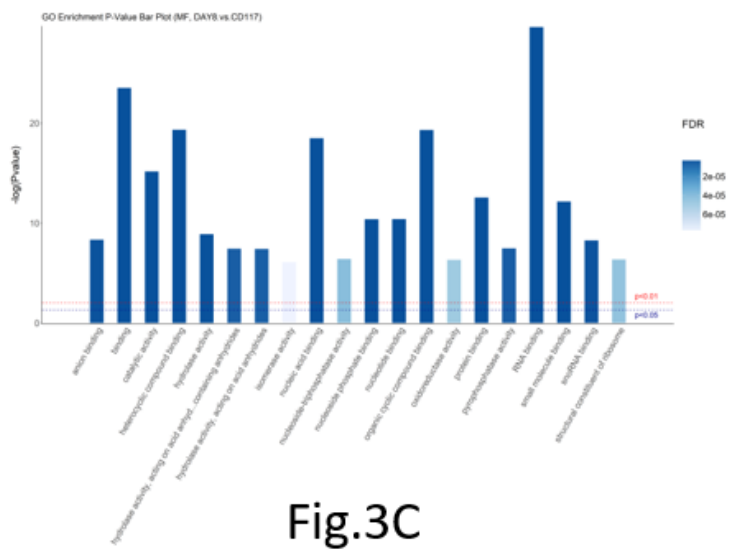

Figure 3

Go functional annotation of DEPs The x-axis shows $\mathrm{GO}$ classification, and the $y$-axis indicates corresponding p-values (A).BP(biological process); (B).CC (cell -ular component); (C).MF(molecular function). 


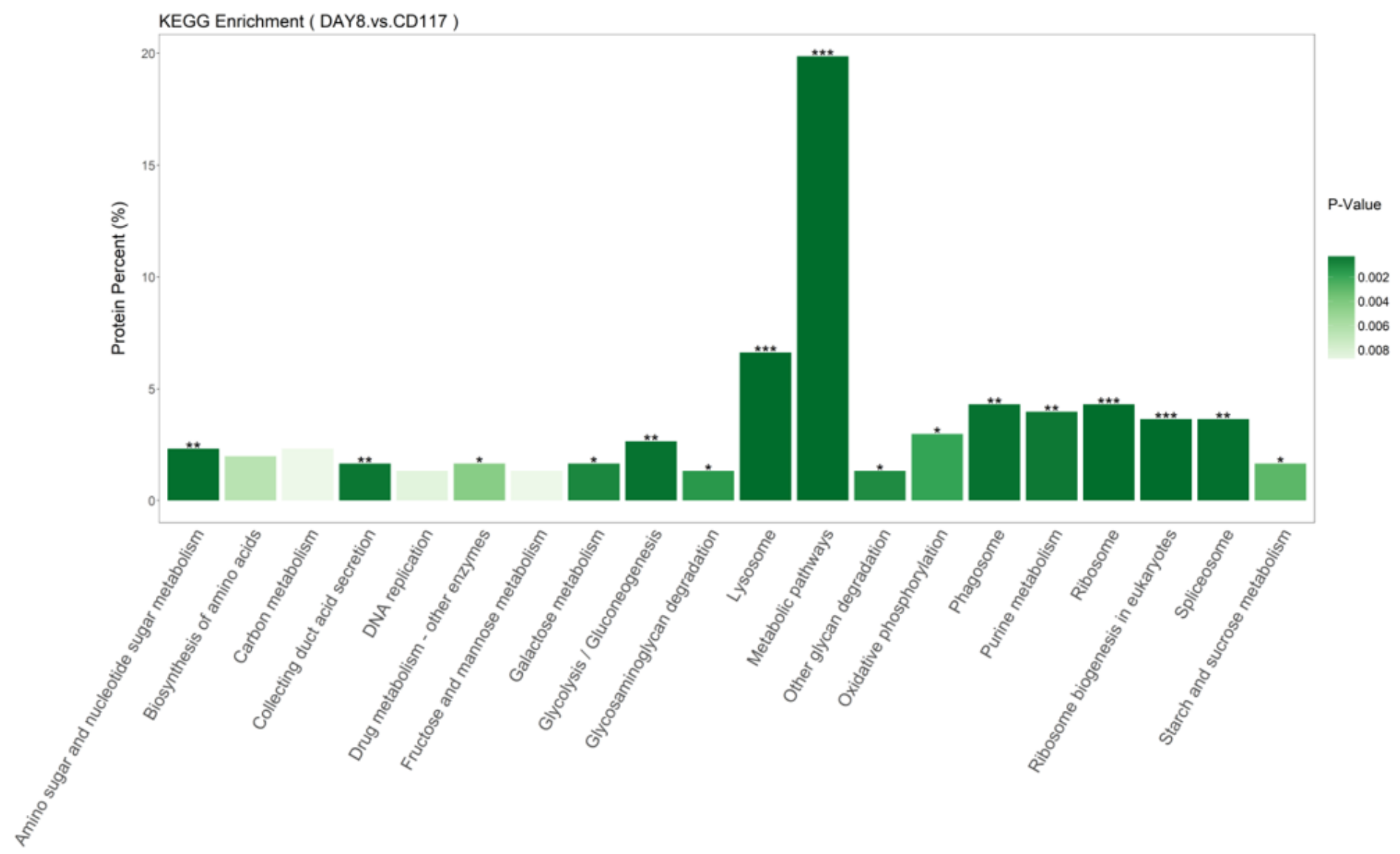

Figure 4

The top20 representative KEGG pathway enrichment analysis of the DEPs The $x$-axis shows representative enriched KEGG pathways, and the $y$-axis indicates corresponding protein percent $(\%)$. 


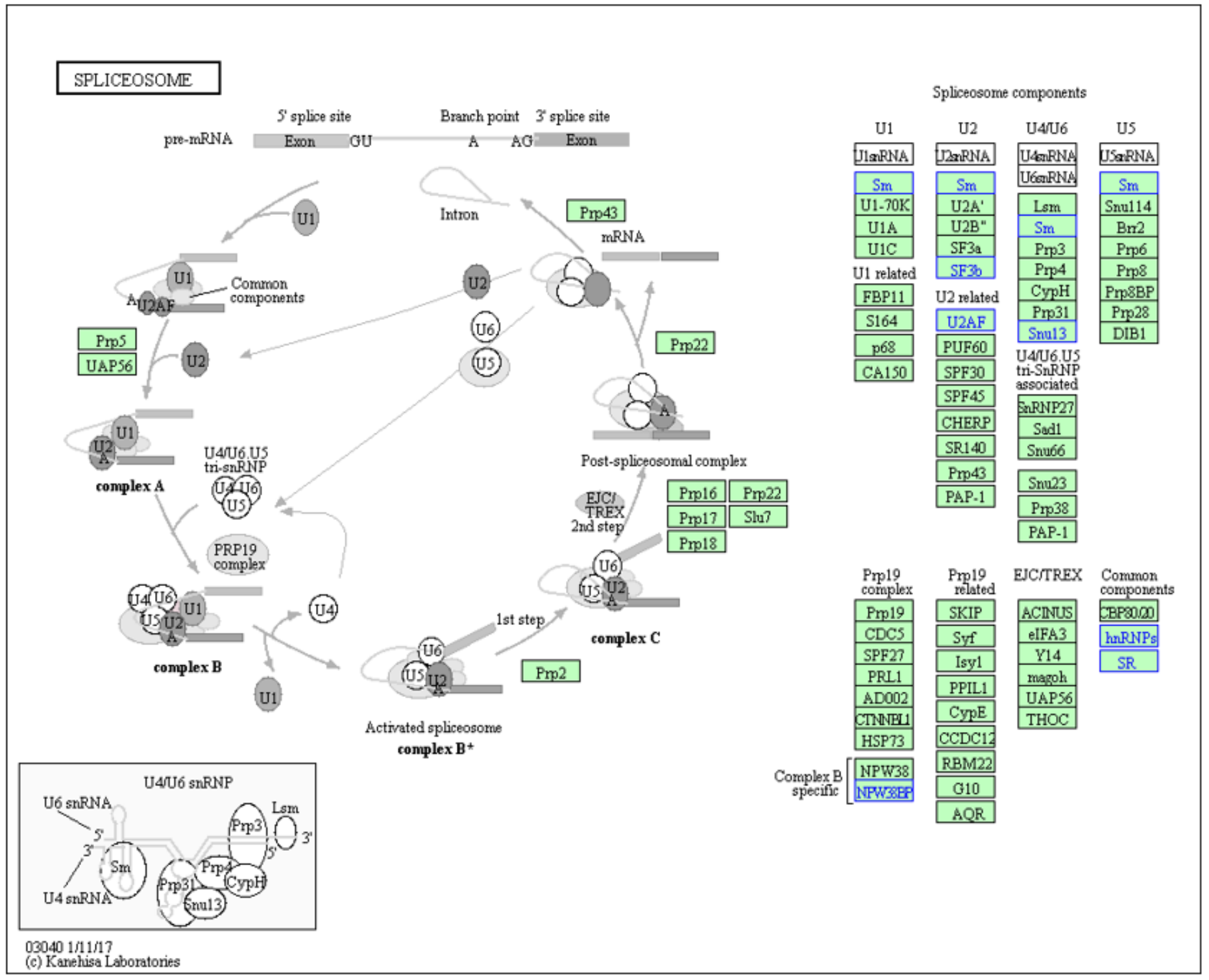

Figure 5

Representative KEGG pathway for mRNA splicing Blue signifies down- regulated proteins related to mRNA splicing in aging group compared with young group. 


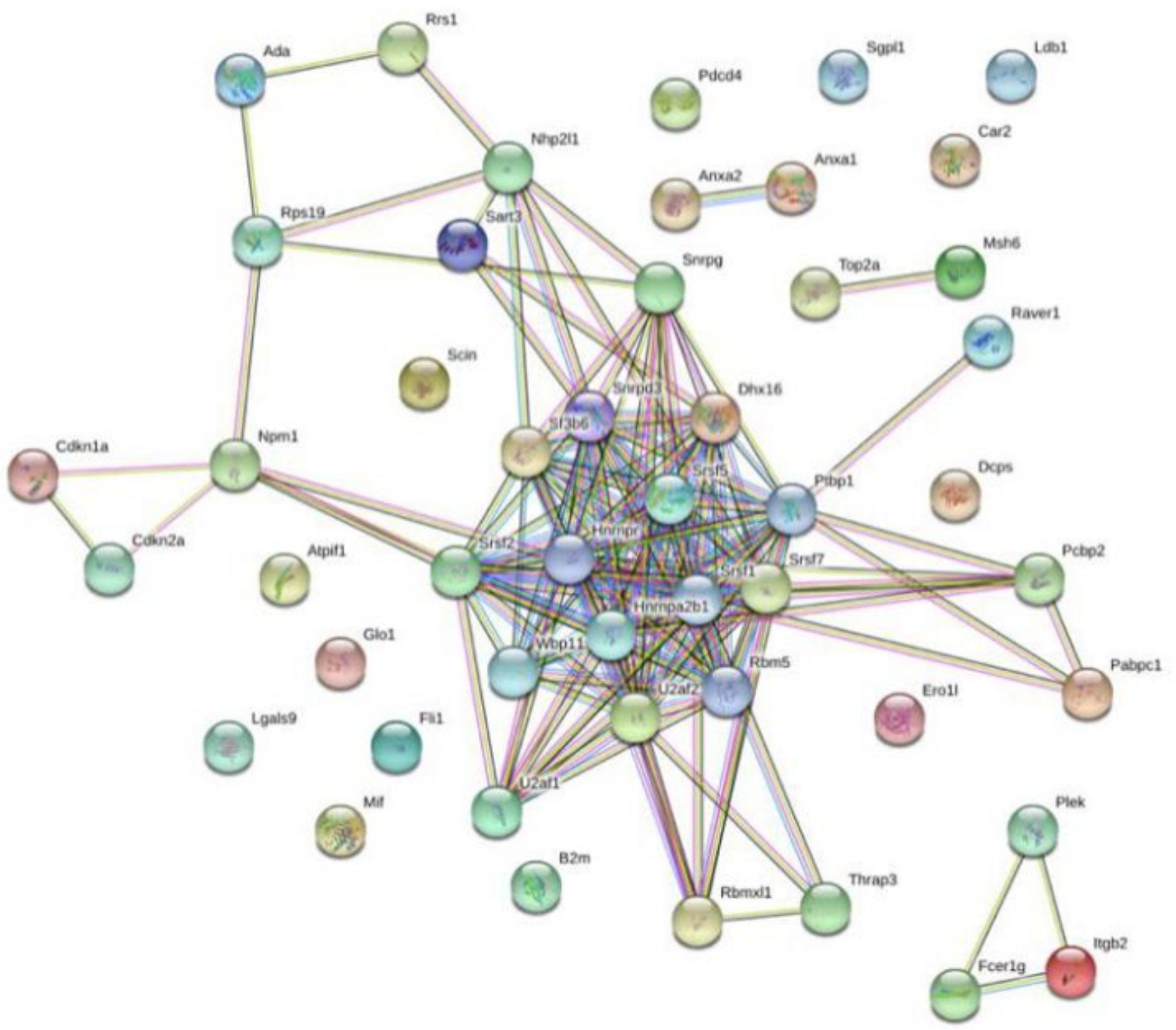

Figure 6

Interaction network of the DEPs involved in mRNA splicing, aging and hemopoiesis 


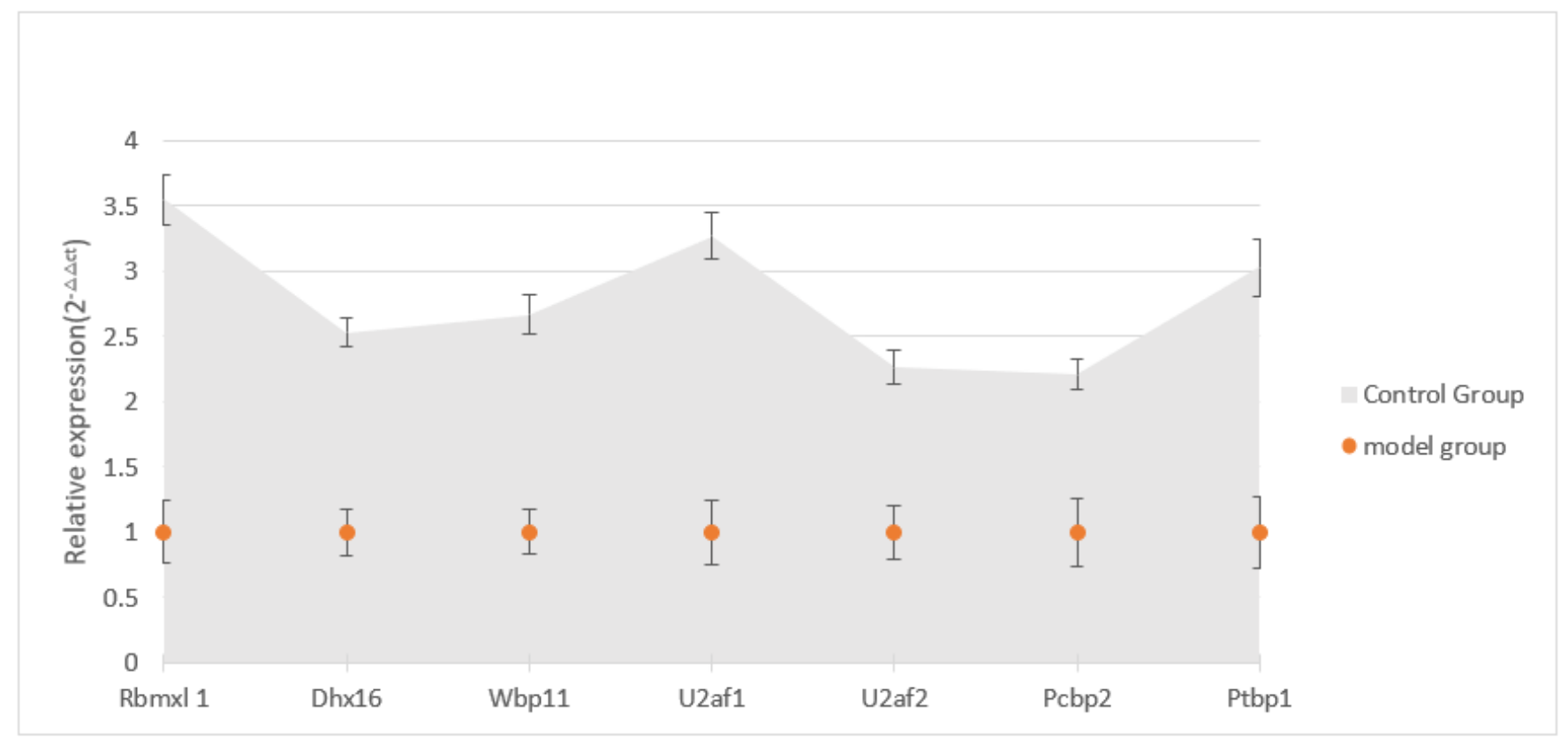

Figure 7

qRT-PCR analysis of representative proteins related to mRNA splicing

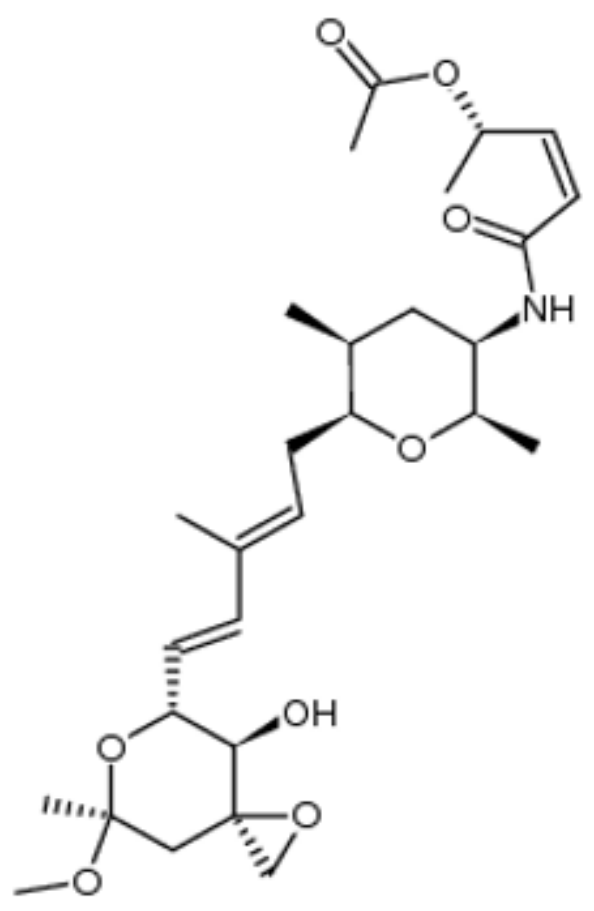

Figure 8 
SSA

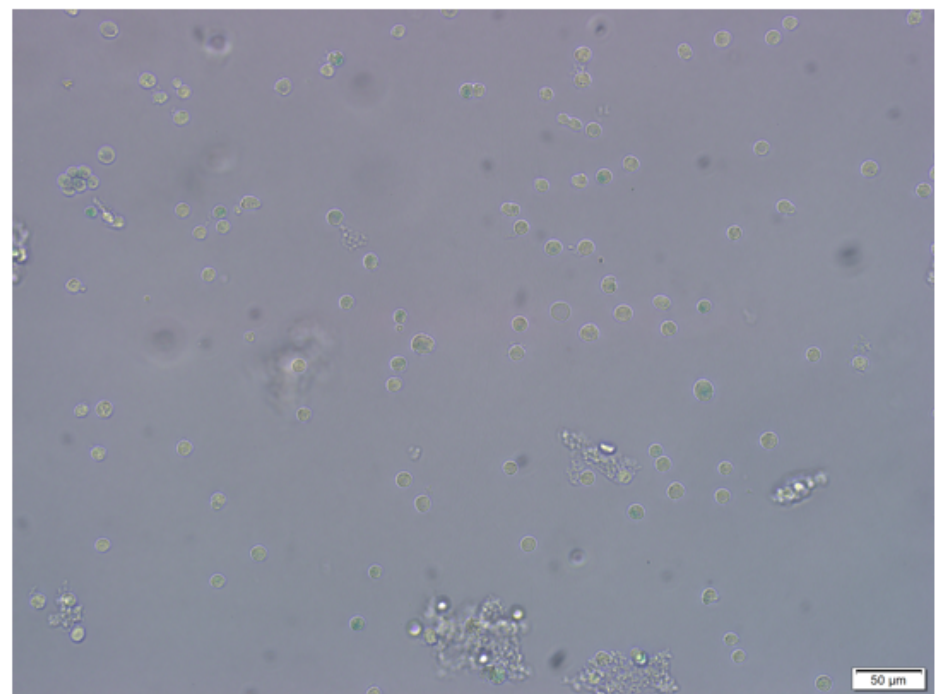

Fig.9A

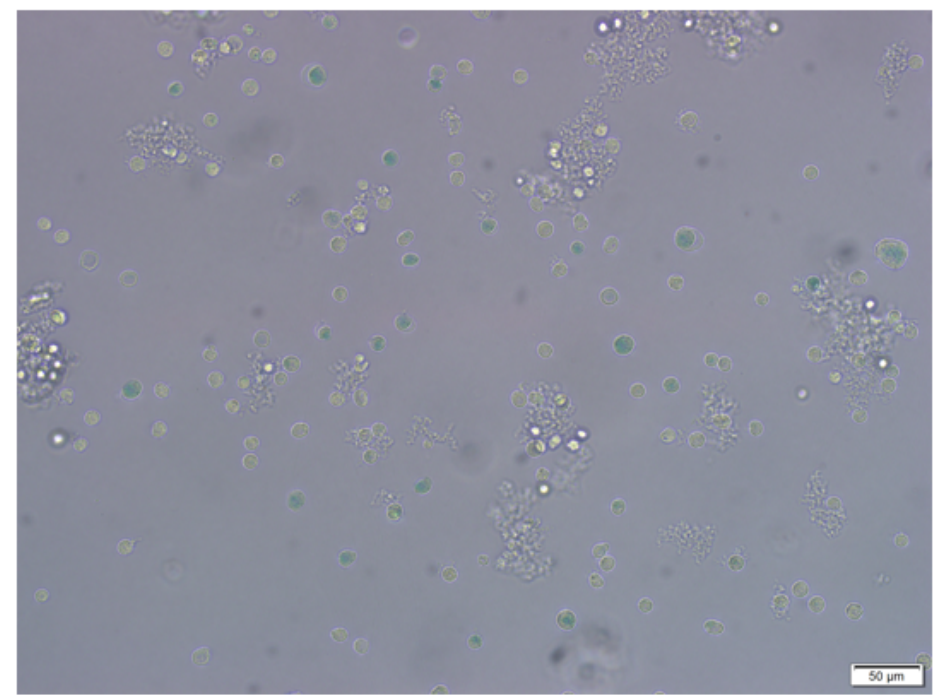

Fig.9B

\section{Figure 9}

Photomicrographs of SA- $\beta$-gal staining $(\times 200)$ The percentage of SA- $\beta$-gal stain-positive cells was significantly increased in SSA group compared with the control group, $n=10$. 


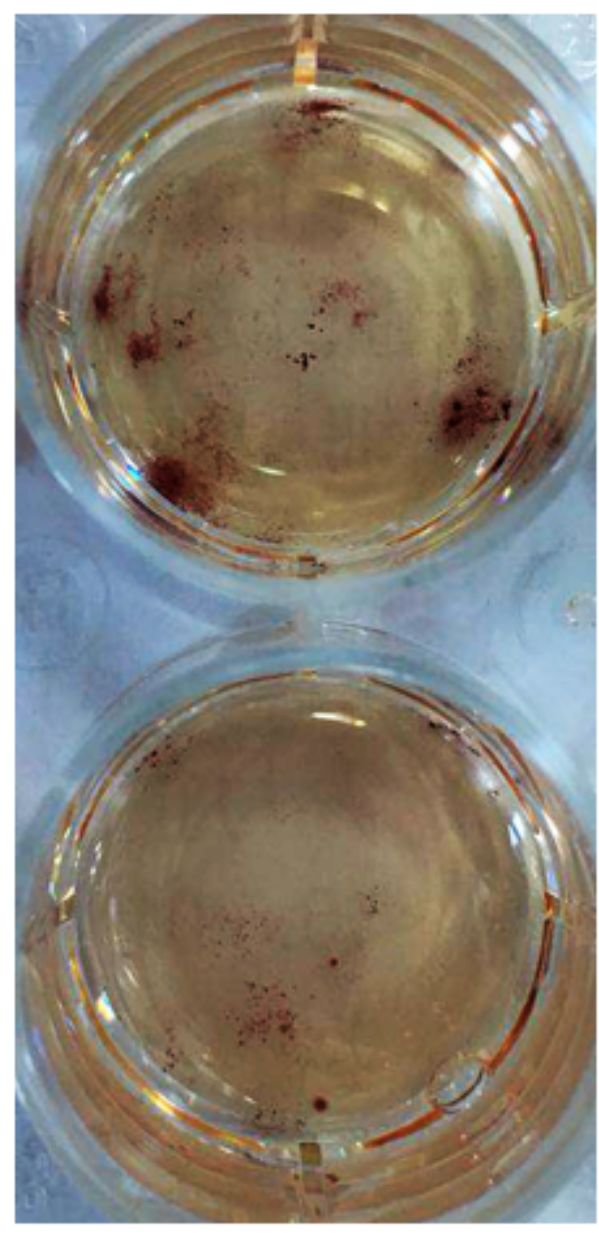

\section{$\leftarrow$ Control group}

\section{$\leftarrow$ SSA group}

\section{Figure 10}

Photomicrographs of CFU-Mix The size and number of CFU-Mix significantly decreased in SSA group compared with young group.

\section{Supplementary Files}

This is a list of supplementary files associated with this preprint. Click to download.

- NC3RsARRIVEGuideline191211.pdf

- supplementFig.docx

- supplementTable.S2.xlsx

- supplementTable.S1.xlsx 